\section{Associação das condições sociais e econômicas com a incidência dos problemas com o sono durante a pandemia de COVID-19}

\author{
Association of social and economic conditions \\ with the incidence of sleep disorders during the \\ COVID-19 pandemic
}

\author{
Asociación de las condiciones sociales y \\ económicas con la incidencia de problemas con \\ el sueño durante la pandemia de COVID-19
}

\author{
Margareth Guimarães Lima 1 \\ Marilisa Berti de Azevedo Barros 1 \\ Celia Landmann Szwarcwald 2 \\ Deborah Carvalho Malta 3 \\ Dalia Elena Romero 2 \\ André Oliveira Werneck 4 \\ Paulo Roberto Borges de Souza Júnior 2
}

doi: 10.1590/0102-311X00218320

\begin{abstract}
Resumo
O sono é importante para a manutenção da saúde física, emocional e para o bem-estar. Poucos estudos avaliaram o efeito das condições socioeconômicas no sono no período da COVID-19. O objetivo foi analisar o aumento ou a incidência dos problemas do sono segundo condições demográficas e econômicas, prévias à pandemia, e segundo mudanças nas condições financeiras, ocupacionais e tarefas domésticas durante a pandemia. Estudo realizado via web, usando dados de 24 de abril a 24 de maio, com 45.160 brasileiros (18 ou mais), com amostra ponderada pelos dados da Pesquisa Nacional por Amostra de Domicílios (PNAD). Mudança na qualidade do sono (desfecho), renda mensal, efeito na renda familiar, na ocupação/trabalho, sexo, faixa etária, situação conjugal e alteração no trabalho doméstico (exposições) foram reportados. Estimamos os percentuais de início ou aumento dos problemas com o sono e

Correspondência

M. G. Lima

Faculdade de Ciências Médicas, Universidade Estadual de Campinas.

Rua Tessália Vieira de Camargo 126, Cidade Universitária, Campinas, SP 13083-887, Brasil.

margarethglima@gmail.com

\footnotetext{
${ }_{1}^{1}$ Faculdade de Ciências Médicas, Universidade Estadual de Campinas, Campinas, Brasil.

2 Instituto de Comunicação e Informação Científica e Brasil.

${ }^{3}$ Escola de Enfermagem, Universidade Federal de Minas Gerais, Belo Horizonte, Brasil.

4 Faculdade de Saúde Pública, Universidade de São Paulo, São Paulo, Brasil.
}

Tecnológica em Saúde, Fundação Oswaldo Cruz, Rio de Janeiro,
\end{abstract} os OR ajustados. A chance de exacerbação dos problemas com o sono foi de $34 \%, 71 \%$ e duas vezes maior nas pessoas com renda inferior a um salário mínimo antes da pandemia, nas que perderam o emprego e naquelas que tiveram a renda muito diminuída/ficaram sem renda, respectivamente. A chance de piorar os problemas do sono foi $82 \%$ maior nas mulheres; três vezes maior $(O R=3,14)$ na população com 18 a 29 anos, em relação aos idosos; e maior com o incremento da quantidade de tarefas domésticas $(O R=2,21)$. Fatores financeiros e ocupacionais foram determinantes na deterioração da qualidade do sono autorreferida, demandando ações rápidas sobre essas condições a fim de minimizar esse impacto. Gênero, faixa etária e rotinas domésticas também merecem atenção em relação à qualidade do sono.

Sono; Classe Social; Infecções por Coronavírus 


\section{Introdução}

Após sete meses da epidemia da doença do novo coronavírus (COVID-19), iniciada na China em dezembro de 2019, o número de casos, no Brasil, atingiu 1.603,055 em 7 de julho de 2020, e 64.867 pessoas foram a óbito até essa data 1.

Para tentar diminuir a rápida propagação do vírus, evitar colapsos no sistema de saúde e até que as intervenções farmacológicas estejam seguramente disponíveis, medidas de distanciamento social foram postas em prática em várias regiões do planeta. Essas medidas incluem o isolamento social, a permanência nas residências, o fechamento de escolas/universidades, a suspensão e a flexibilização de comércio, a restrição de transporte e o cancelamento de encontros sociais 2,3. As medidas de distanciamento social, em várias regiões do Brasil, foram implementadas a partir da Portaria no 356, de 11 de março de 2020 4, do Ministério da Saúde.

As estratégias de distanciamento social podem impactar várias dimensões da vida, tais como o trabalho e a ocupação, os rendimentos familiares e as relações sociais 5,6,7,8. Diante dessas condições, juntamente com o medo de contrair a doença e a exposição a uma quantidade de notícias 9,10 , a saúde emocional 8,11,12 e aspectos relacionados, como a qualidade do sono, tendem a estar deteriorados.

$\mathrm{O}$ sono de boa qualidade tem forte relação com as questões emocionais, com a saúde física e com o bem-estar 13,14,15, atuando nos sistemas imune, metabólico, endócrino e nos processos inflamatórios 16 . Autores alertam que o sono ruim pode estar relacionado com a suscetibilidade à infecção por COVID-19 17,18. Estudos em diferentes países têm encontrado alta prevalência da qualidade do sono ruim durante a pandemia, chegando a taxas de $57 \%$, na Itália 12 .

Pesquisas conduzidas para investigar os fatores associados à qualidade do sono durante a pandemia, na população geral, trazem informações sobre a forte relação com as questões emocionais e o estresse 10,12,19,20,21,22. Além disso, poucas avaliaram as questões sociais 8,12,22, sobretudo as condições econômicas, impactando a qualidade do sono nesse período, e, de acordo com nossa revisão, não foi avaliado o efeito da perda da renda familiar na incidência de problemas com o sono. Nesse sentido, nosso objetivo foi analisar o relato do aumento ou da incidência dos problemas do sono na população adulta brasileira segundo condições demográficas e socioeconômicas, prévias à pandemia, e segundo as mudanças nas condições financeiras, ocupacionais e carga de tarefas domésticas durante a pandemia.

\section{Métodos}

Nós utilizamos dados da ConVid - Pesquisa de Comportamentos, conduzida no Brasil pela Fundação Oswaldo Cruz (Fiocruz), em parceria com a Universidade Estadual de Campinas (Unicamp) e a Universidade Federal de Minas Gerais (UFMG), no período de 24 de abril a 24 de maio. O projeto foi aprovado pelo Comitê Nacional de Ética em Pesquisa sob processo no 3.980.277. O inquérito foi conduzido por meio de um questionário autopreenchido, via web, com a finalidade de avaliar mudanças ocorridas nas condições de vida, nas atividades de rotina, na saúde e no acesso aos serviços de saúde, no estilo de vida e nos aspectos emocionais da população vivendo em território brasileiro durante a pandemia do novo coronavírus. O questionário foi elaborado com a utilização do aplicativo REDCap (Research Electronic Data Capture - https://www.redcapbrasil.com.br/) que consiste numa plataforma digital de coleta, gerenciamento e disseminação de dados. Os dados foram armazenados em servidores da Fiocruz.

A amostra foi obtida por procedimento de amostragem em cadeia em que, no primeiro estágio, os pesquisadores do estudo convidaram 20 outros pesquisadores em diferentes regiões do Brasil, chamados de influenciadores, que enviaram o link da pesquisa para, pelo menos, 12 pessoas das suas redes sociais, obedecendo a uma estratificação por sexo, faixa de idade (18-39; 40-59; 60+ anos) e grau de escolaridade (Ensino Médio incompleto ou menos; Ensino Médio completo ou mais). A essas pessoas foi solicitado que convidassem outras três pessoas de suas redes sociais e assim por diante. A amostra totalizou 45.161 indivíduos e foi calibrada de acordo com dados da Pesquisa Nacional por Amostra de Domicílios (PNAD 2019), com a finalidade de obter a mesma distribuição por Unidade da Federação (UF), sexo, faixa etária, cor da pele e nível de escolaridade da população brasileira. Detalhes sobre o método e o procedimento amostral foram publicados em outro artigo 23. 
O desfecho estudado foi o relato do aumento dos problemas de sono entre os indivíduos que já se queixavam desses agravos ou o relato do início dos problemas com o sono. A variável partiu da seguinte pergunta: "A pandemia afetou a qualidade do seu sono?", com as seguintes categorias de resposta: (1) "Não afetou nada, continuo dormindo bem"; (2) "Com a pandemia comecei a ter problemas com o sono”; (3) "Eu já tinha problemas de sono e eles continuaram da mesma forma”; (4) "Eu já tinha problemas de sono e eles pioraram bastante"; (5) "Eu já tinha problemas de sono, mas eles diminuíram". As categorias 1, 3 e 5 foram agregadas, compondo o grupo (1) dos que não tiveram aumento ou início dos problemas com o sono, e as categorias 2 e 4 foram agregadas, compondo o grupo (2) dos indivíduos que relataram início ou aumento dos problemas com o sono.

As variáveis de exposição foram: sexo, faixa etária (18-29, 30-49, 50-59 e 60+ anos), número de moradores no domicílio ( 1 a 3, 4, 5 ou mais), vive com cônjuge ou companheiro, nível de escolaridade (Ensino Fundamental completo ou menos, Ensino Médio completo, Superior completo ou mais), renda mensal familiar per capita em salários mínimos $(<1,1<2,2<4,4$ ou mais), efeito na renda familiar no período estudado (não diminuiu, diminuiu um pouco, diminuiu muito ou ficou sem renda), efeito na ocupação no período estudado (não perdeu o emprego, perdeu o emprego ou ficou sem trabalho), alteração na quantidade e tipo de trabalho doméstico (não aumentou, aumentou um pouco, aumentou muito).

As análises consistiram em estimativas de prevalências e intervalos de 95\% de confiança (IC95\%) da piora ou início dos problemas com o sono durante a pandemia segundo as variáveis de exposição. Também foram estimados odds ratios (OR) e IC95\% por modelo de regressão logística múltipla. Todas as análises foram ajustadas por sexo e idade. Na análise com número de moradores no domicílio, foi acrescentada a variável renda mensal per capita para os ajustes.

As análises foram conduzidas utilizando o software estatístico Stata 15.0 (https://www.stata.com), e foi considerado o peso amostral da pós-estratificação.

\section{Resultados}

Foram estudados 45.160 indivíduos com 18 anos ou mais. A renda familiar diminuiu muito ou zerou, durante a pandemia, para $31,8 \%$ da população, e $26,6 \%$ das pessoas ficaram sem trabalhar ou perderam o emprego. Entre as pessoas que relataram diminuição extrema da renda, 39,8\% apresentavam rendimento mensal prévio menor do que 1 salário mínimo, enquanto 19,8\% possuíam renda de 4 salários mínimos ou mais. O percentual de perda de ocupação foi de $29 \%$ nos participantes de menor renda. O aumento demasiado do trabalho doméstico foi referido por $20,2 \%$ da população, com percentual duas vezes maior no sexo feminino ( $\mathrm{p}<0,001)$ (dados não apresentados em tabela).

$\mathrm{Na}$ análise bivariada, considerando os fatores sociais e econômicos prévios à pandemia, o aumento ou a incidência dos problemas com o sono foi referida, em maior percentual, pelas mulheres, pelos mais jovens, por aqueles que convivem com mais pessoas no domicílio, que não vivem com cônjuge ou companheiro e com menor rendimento mensal per capita (Tabela 1).

A piora ou incidência de problemas no sono também foi maior nas pessoas em que a renda diminuiu muito ou que ficaram sem rendimento durante a pandemia, nas que perderam o emprego ou ficaram sem trabalhar e entre as que relataram pouco $(47,4 \%)$ ou muito $(58,1 \%)$ aumento na quantidade de trabalho doméstico (Tabela 2). A associação dos problemas do sono com a adição do trabalho doméstico por conta da pandemia não teve interação por sexo, ou seja, associou-se, de maneira similar, na população masculina e feminina (dados não apresentados em tabela).

$\mathrm{Na}$ análise multivariada, os resultados segundo condições financeiras e ocupacionais mostram que a chance de exacerbação dos problemas com o sono foi, respectivamente, $34 \%$ e $71 \%$ maior nas pessoas com renda mensal per capita inferior a 1 salário mínimo antes da pandemia e nas que perderam o emprego. Nos indivíduos que tiveram a renda muito diminuída ou ficaram sem renda, a chance de alterações no sono foi duas vezes maior $(\mathrm{OR}=2,08)$. Nas mulheres, a chance da incidência dos problemas com o sono foi de $82 \%$; aumentou com gradiente, conforme diminuição da idade, com o OR crescendo de 1,72 entre pessoas com 50-59 anos, para 3,14, no subgrupo de 18-29 anos, em relação aos idosos. Aumento na quantidade de tarefas domésticas ocasionou em $52 \%$ a chance de aumentar ou iniciar os problemas com o sono, mas o maior incremento da quantidade de tarefas domésticas dobrou a chance $(\mathrm{OR}=2,21)$ (Tabela 3$)$. 
Tabela 1

Fatores anteriores à pandemia de COVID-19, preditores da incidência ou aumento dos problemas com o sono durante a pandemia.

\begin{tabular}{|c|c|c|c|c|}
\hline & \multirow[t]{2}{*}{ n } & \multicolumn{3}{|c|}{ Aumento ou incidência dos problemas com o sono } \\
\hline & & $\%$ & IC95\% & Valor de $p$ \\
\hline Escolaridade & & & & 0,2995 \\
\hline Ensino Fundamental completo ou menos & 5.024 & 42,4 & $37,3-47,6$ & \\
\hline Ensino Médio completo & 32.701 & 45,4 & $43,6-47,3$ & \\
\hline Superior completo ou mais & 7.436 & 44,2 & $43,5-44,9$ & \\
\hline Renda familiar mensal per capita anterior à pandemia (salários mínimos) & & & & $<0,001$ \\
\hline$<1$ & 20.996 & 50,4 & $48,2-52,7$ & \\
\hline $1<2$ & 10.409 & 42,0 & $39,0-45,1$ & \\
\hline $2<4$ & 7.282 & 39,8 & $36,8-42,8$ & \\
\hline 4 ou mais & 3.697 & 36,5 & $33,4-39,7$ & \\
\hline Sexo & & & & $<0,001$ \\
\hline Masculino & 20.975 & 37,7 & $35,3-40,2$ & \\
\hline Feminino & 24.186 & 51,1 & $49,5-52,7$ & \\
\hline Faixa etária (anos) & & & & $<0,001$ \\
\hline $18-29$ & 9.173 & 55,0 & $52,3-57,6$ & \\
\hline $30-49$ & 7.190 & 48,2 & $45,8-52,7$ & \\
\hline $50-59$ & 17.656 & 41,1 & $38,0-44,2$ & \\
\hline 60 & 11.142 & 29,2 & $26,2-32,4$ & \\
\hline Número de moradores no domicílio & & & & 0,0001 \\
\hline $1-3$ & 28.972 & 42,3 & $40,6-44,1$ & \\
\hline 4 & 9.638 & 48,5 & $45,5-51,4$ & \\
\hline 5 ou mais & 6.465 & 51,3 & $46,7-55,8$ & \\
\hline Vive com cônjuge & & & & $<0,001$ \\
\hline Não & 20.989 & 49,1 & $47,1-51,1$ & \\
\hline Sim & 24.084 & 41,2 & $39,2-43,3$ & \\
\hline
\end{tabular}

IC95\%: intervalo de 95\% de confiança.

\section{Tabela 2}

Incidência de problemas com o sono segundo mudanças econômicas, ocupacionais e trabalho doméstico, ocorridos durante a pandemia de COVID-19.

\begin{tabular}{|c|c|c|c|c|}
\hline \multirow[t]{2}{*}{ Condições sociais } & \multirow[t]{2}{*}{$\mathbf{n}$} & \multicolumn{3}{|c|}{ Aumento ou incidência de problemas com o sono } \\
\hline & & $\%$ & IC95\% & Valor de $p$ \\
\hline Efeito na renda per capita da família & & & & $<0,001$ \\
\hline Não diminuiu & 17.044 & 37,3 & $35,4-39,4$ & \\
\hline Diminuiu um pouco & 13.667 & 42,7 & $40,6-45,3$ & \\
\hline Diminuiu muito ou ficou sem renda & 14.333 & 56,1 & $53,3-58,9$ & \\
\hline Efeito na ocupação/trabalho & & & & $<0,001$ \\
\hline Não perdeu o emprego & 34.098 & 42,2 & $40,6-43,8$ & \\
\hline Perdeu o emprego ou ficou sem trabalho & 10.546 & 54,7 & $51,5-57,8$ & \\
\hline Alteração na quantidade e tipo de trabalho doméstico & & & & $<0,001$ \\
\hline Não aumentou & 17.208 & 35,6 & $33,4-37,9$ & \\
\hline Aumentou um pouco & 18.652 & 47,4 & $45,2-49,6$ & \\
\hline Aumentou muito & 9.069 & 58,1 & $55,1-61,0$ & \\
\hline
\end{tabular}

IC95\%: intervalo de 95\% de confiança. 
Tabela 3

Associação de condições prévias à pandemia de COVID-19 e mudanças sociais e econômicas associadas à incidência e ao aumento dos problemas com o sono durante a pandemia.

\begin{tabular}{|c|c|}
\hline Variáveis & OR ajustado (IC95\%) \\
\hline \multicolumn{2}{|l|}{ Escolaridade } \\
\hline Ensino Fundamental completo ou menos & 1,00 \\
\hline Ensino Médio completo & $0,95(0,74-1,20)$ \\
\hline Superior completo ou mais & $0,85(0,68-1,07)$ \\
\hline \multicolumn{2}{|c|}{ Renda familiar mensal per capita anterior à pandemia (salários mínimos) } \\
\hline$<1$ & 1,00 \\
\hline $1<2$ & $1,06(0,88-1,28)$ \\
\hline $2<4$ & $1,10(0,91-1,32)$ \\
\hline 4 ou mais & $1,34(1,13-1,59)$ \\
\hline \multicolumn{2}{|l|}{ Efeito na renda per capita da família } \\
\hline Não diminuiu & 1,00 \\
\hline Diminuiu um pouco & $1,12(0,97-1,29)$ \\
\hline Diminuiu muito ou ficou sem renda & $2,08(1,80-2,40)$ \\
\hline \multicolumn{2}{|l|}{ Efeito na ocupação/trabalho } \\
\hline Não perdeu o emprego & 1,00 \\
\hline Perdeu o emprego ou ficou sem trabalho & $1,71(1,48-1,98)$ \\
\hline \multicolumn{2}{|l|}{ Sexo } \\
\hline Masculino & 1,00 \\
\hline Feminino & $1,82(1,61-2,07)$ \\
\hline \multicolumn{2}{|l|}{ Faixa etária (anos) } \\
\hline $18-29$ & $3,14(2,60-3,80)$ \\
\hline $30-49$ & $2,35(1,96-2,82)$ \\
\hline $50-59$ & $1,72(1,41-2,11)$ \\
\hline 60 & 1,00 \\
\hline \multicolumn{2}{|l|}{ Número de moradores no domicílio } \\
\hline $1-3$ & 1,00 \\
\hline 4 & $1,01(0,87-1,17)$ \\
\hline 5 ou mais & $1,21(0,97-1,50)$ \\
\hline \multicolumn{2}{|l|}{ Vive com cônjuge } \\
\hline Não & $1,13(1,00-1,29)$ \\
\hline Sim & 1,00 \\
\hline \multicolumn{2}{|c|}{ Alteração na quantidade e tipo de trabalho doméstico } \\
\hline Não aumentou & 1,00 \\
\hline Aumentou um pouco & $1,52(1,33-1,74)$ \\
\hline Aumentou muito & $2,21(1,87-2,61)$ \\
\hline
\end{tabular}

IC95\%: intervalo de 95\% de confiança; OR: odds ratio.

Nota: as análises foram ajustadas por sexo e faixa etária. A variável renda foi acrescentada para o ajuste na associação com número de moradores. Valores em negrito representam $p<0,05$. 


\section{Discussão}

Pelo nosso conhecimento, este é o primeiro estudo no Brasil e na América Latina que avaliou o relato de problemas com o sono durante a pandemia de COVID-19, de acordo com condições sociais e econômicas. Vale mencionar que as pesquisas sobre sono, nesse período, são mais frequentes na China 8,19,20,24 e em países desenvolvidos 6,10,12,21,22 com lacunas de pesquisas representativas de outros países afetados pela pandemia, principalmente nos de baixa ou média renda. Constatamos que fatores financeiros e ocupacionais foram determinantes na deterioração da qualidade do sono autorreferida.

A renda prévia à pandemia foi preditora do aumento dos problemas com o sono. A chance desses problemas foi maior no estrato de indivíduos com rendimento menor do que 1 salário mínimo, em relação àqueles com quatro salários ou mais. Em estudos anteriores a COVID-19, autores têm identificado o efeito do nível socioeconômico sobre o sono 25 , mas, segundo pesquisas de base populacional realizadas com adultos em município brasileiro 15,26 , os proventos mensais não se associaram com a qualidade ou a duração do sono. A baixa renda pode não interferir na duração do sono, no período avaliado, mas pode predizer uma piora da qualidade do sono em situações adversas e, especialmente, nesse período de pandemia e de incertezas. Além da renda prévia, ter os rendimentos diminuídos ou ficar sem renda, nesse período, aumentou a chance de ter problemas com o sono em $69 \%$. Vale salientar que, no nosso estudo, o prejuízo de renda no período da pandemia foi duas vezes maior nos mais pobres. A perda do trabalho também foi um dos determinantes do aumento dos problemas com a qualidade do sono e foi maior também entre os indivíduos situados no estrato de menor rendimento mensal. Esses resultados alertam para o problema das desigualdades socioeconômicas no Brasil, dificultando o enfrentamento da pandemia de maneira equânime.

A Comissão Econômica para a América Latina e o Caribe (CEPAL), em estudo recente comparando o período 2008/2014 com 2014/2018, aponta o Brasil, entre os países de sua região, em piores condições em relação à pobreza, à desigualdade e ao desemprego 27. Desde 2014, a taxa de desemprego vem aumentando no Brasil, subindo de 6,8\%, nesse ano, para 12,8\%, em 2017. Em 2019, o desemprego esteve em 11,9\%. Esse panorama atingiu, em maior parte, a parcela mais pobre da população, que também sofreu com a variação da renda nesse período 28,29. Provavelmente, a partir de 2017, devido a alterações na legislação trabalhista, a classe dos trabalhadores informais e dos autônomos cresceu bastante, com estimativas de 38 milhões de trabalhadores atuando nesses setores 26 . As mulheres, os menos escolarizados, os trabalhadores informais e os mais pobres parecem estar vinculados a ocupações mais vulneráveis durante a pandemia, ou seja, trabalhos que podem ser diretamente afetados nesse período por terem interação direta com o cliente 30. A desigualdade social, a crise financeira e as condições trabalhistas apontam que o brasileiro não estava em condições para enfrentar a crise socioeconômica suscitada pela COVID-19.

A estratégia do pagamento de um auxílio emergencial de BRL 600,00, que representa cerca de 58\% do salário mínimo mensal, para trabalhadores informais e autônomos, foi implementada em 9 de abril e pode contribuir para melhorar a situação entre os mais pobres. No entanto, esse auxílio demorou para chegar até a população e foi de difícil acesso, principalmente entre a população menos favorecida. Também, as pessoas aptas a receber o auxílio podem ter se deparado com sentimento de angústia pelas incertezas de recebimento nos próximos meses.

As crises econômicas e o desemprego são fatores que afetam diretamente a saúde mental da população 31,32, o que pode incidir nos problemas do sono, principalmente no distúrbio de insônia 33. As medidas em prol de minimizar os problemas financeiros da população mais pobre tornam-se importantes nesse período para evitar ou diminuir os possíveis efeitos nos problemas relacionados ao sono.

Ser mulher e adulto jovem aumentou a chance de exacerbação dos problemas com o sono durante a pandemia. Esses resultados estão de acordo com estudo conduzido na Itália e na Espanha, que apresentou maior risco de desenvolver distúrbio do sono nesses subgrupos da população 12,22. Já em estudo realizado na China, mesmo na análise bruta, essas associações foram observadas, possivelmente, porque a estratificação por faixa etária considerou apenas pessoas com menos e mais de 35 anos 8 . Uma explicação plausível para essa questão seria a rotina diária alterada nos adultos mais jovens, mudando os horários de ir para a cama e de levantar ${ }^{34}$. Também vale considerar a questão do estresse emocional de passar por confinamento em fase de grandes aspirações na vida, como estudos, início de carreira, casamento. Os encontros sociais, que são mais frequentes nessa fase, também ficaram 
comprometidos. Já na etapa da velhice, a solidão, o distanciamento social e os problemas de sono são estruturais, que acompanham a complexidade do envelhecimento 35. Embora seja menos intensa a declaração de piora do sono nos idosos, durante a pandemia, se comparado com os jovens, as consequências dos efeitos podem ser fatais 36 .

Especialistas em medicina do sono, componentes da Sociedade de Medicina dos Comportamentos do Sono (SBSM), fizeram uma força-tarefa para elaborar dicas sobre como lidar melhor com o sono diante dos desafios da pandemia de COVID-19. Entre elas, evitar atividades no quarto ou ficar na cama quando não estiver dormindo; acordar e sair da cama, independente das obrigações diárias; inserir rotinas de horários para dormir para crianças e adolescentes 34 .

As mulheres tendem a relatar piores condições de saúde emocional 37, inclusive no período da pandemia 38 , o que pode estar atrelado ao relato da pior qualidade do sono. Também vale considerar a dupla jornada de trabalho, que pode estar mais forte nesse período, principalmente em relação às tarefas domésticas, levando a questões de cansaço, nervosismo, preocupação e até mesmo ocupando maior tempo, limitando ou piorando a qualidade do sono no sexo feminino. No nosso estudo, verificamos que o aumento demasiado das tarefas domésticas foi duas vezes maior nas mulheres em relação aos homens.

O alto volume da rotina doméstica, neste estudo, associou-se, com grande magnitude, aos problemas do sono. A chance de iniciar ou agravar distúrbios do sono foi duas vezes maior com o incremento da carga de trabalho doméstico. A execução de tarefas domésticas tende a se associar com desfechos de pior saúde emocional 39, e autores têm encontrado associações dessas atividades com dores nas costas 40,41 que podem contribuir para piorar a qualidade do sono. Também é possível que o aumento das atividades domésticas contribua para aumentar o tempo para execução das tarefas diárias totais, privando as pessoas do tempo de sono. Além disso, a carga doméstica aumentada e a execução de tarefas que não eram desempenhadas anteriormente, como cozinhar, cuidar de idosos, higienizar compras, podem ter gerado preocupações e estresse no dia a dia.

Ao considerar os resultados do estudo, é necessário avaliar alguns aspectos. O período analisado corresponde ao início das estratégias de distanciamento social e é possível que o impacto seja diferente em outra fase. A pergunta utilizada para analisar a alteração do sono é única e não se refere a uma escala já utilizada. No entanto, é um relato da alteração do sono. Em estudo brasileiro utilizando uma única pergunta para detectar fatores associados à qualidade do sono, autores observaram que a autoavaliação do sono apresentou fortes associações com queixas do sono 26 . Outros autores têm utilizado questões únicas, autorreferidas, para analisar a qualidade do sono 42 , e torna-se difícil, em pesquisas realizadas via $w e b$, inserir instrumentos padronizados, aumentando o tempo de resposta e diminuindo a adesão à participação. Também é necessário considerar que a questão do sono se utiliza de uma comparação, do próprio sujeito, do período prévio à pandemia para após, que pode inserir um viés de memória, embora o prazo para lembrança seja curto. Além disso, devido ao próprio delineamento da pesquisa, é possível que grupos populacionais estejam sub-representados como as pessoas sem acesso à Internet. As forças do estudo se referem à informação que ele fornece em relação ao impacto das condições socioeconômicas no aumento dos problemas com o sono durante a pandemia de COVID-19, considerando que as pesquisas nesse tema são escassas. A amostra foi composta por 45.161 indivíduos e calibrada com pesos baseados na PNAD.

Os resultados do estudo revelaram que os mais pobres e os que perderam a renda e o trabalho no período da pandemia, as mulheres, os mais jovens, os indivíduos que não vivem com cônjuge ou companheiro e que tiveram suas tarefas domésticas aumentadas apresentaram maior chance da incidência ou da exacerbação dos problemas com o sono. Esses determinantes devem ser considerados em estratégias para minimizar os efeitos da pandemia e, sobretudo, do distanciamento social, na saúde da população, considerando a importância do sono para manutenção da saúde e do bem-estar. 


\section{Colaboradores}

M. G. Lima contribuiu na concepção do artigo, análise e interpretação dos dados e da redação do artigo. M. B. A. Barros, C. L. Szwarcwald, D. C. Malta e D. E. Romero contribuíram na interpretação dos dados, redação e revisão crítica do manuscrito. A. O. Werneck e P. R. B. Souza Júnior contribuíram na redação e revisão crítica do manuscrito.

\section{Referências}

1. World Health Organization. Coronavirus disease (COVID-19) Situation Report - 169. https://www.who.int/docs/default-source/ coronaviruse/situation-reports/20200707covid-19-sitrep-169.pdf?sfvrsn=c6c69c88_2 (acessado em 09/Jun/2020).

2. Wilder-Smith A, Freedman SO. Isolation, quarantine, social distancing and community containment: pivotal role for old-style public health measures in the novel coronavirus (2019-nCoV) outbreak. J Travel Med 2020; 27:3-13.

3. Garcia LP, Duarte E. Intervenções não farmacológicas para o enfrentamento à epidemia da COVID-19 no Brasil. Epidemiol Serv Saúde 2020; 29:e2020222.

4. Ministério da Saúde. Portaria no 356, de 11 de março de 2020. Dispõe sobre a regulamentação e operacionalização do disposto na Lei no 13.979 , de 6 de fevereiro de 2020, que estabelece as medidas para enfrentamento da emergência de saúde pública de importância internacional decorrente do coronavírus (COVID-19). Diário Oficial da União 2020; 12 mar.

5. Losada-Baltar A, Jiménez-Gonzalo L, GallegoAlberto L, Pedroso-Chaparro MS, FernandesPires J, Márquez-González M. We're staying at home. Association of self-perceptions of aging, personal and family resources and loneliness with psychological distress during the lockdown period of COVID-19. J Gerontol B Psychol Sci Soc Sci 2020; 76:e10-6.

6. Stanton R, To QG, Khalesi S, Williams SL, Alley SJ, Thwaite TL, et al. Depression, anxiety and stress during COVID-19: associations with changes in physical activity, sleep, tobacco and alcohol use in Australian adults. Int J Environ Res Public Health 2020; 17:4065.

7. Lewnard JA, Lo NC. Scientific and ethical basis for social-distancing interventions against COVID-19. Lancet Infect Dis 2020; 20:631-3.

\section{Informações adicionais}

ORCID: Margareth Guimarães Lima (0000-00016996-0745); Marilisa Berti de Azevedo Barros (0000-0003-3974-195X); Celia Landmann Szwarcwald (0000-0002-7798-2095); Deborah Carvalho Malta (0000-0002-8214-5734); Dalia Elena Romero (0000-0002-2643-9797); André Oliveira Werneck (0000-0002-9166-4376); Paulo Roberto Borges de Souza Júnior (0000-0002-8142-4790).

8. Huang Y, Zhao N. Generalized anxiety disorder, depressive symptoms and sleep quality during COVID-19 outbreak in China: a webbased cross-sectional survey. Psychiatry Res 2020; 288:112954.

9. Asmundson GJG, Taylor S. Coronaphobia: fear and the 2019-nCoV outbreak. J Anxiety Disord 2020; 70:102196.

10. Voitsidi P, Gliatas I, Bairachtari V, Papadopoulou K, Papageorgiou G, Parlapani E, et al. Insomnia during the COVID-19 pandemic in a Greek population. Psychiatry Res 2020; 289:113076.

11. Vindegaard N, Eriksen Benros M. COVID-19 pandemic and mental health consequences: systematic review of the current evidence. Brain Behav Immun 2020; 89:531-42.

12. Casagrande M, Favieri F, Tambelli R, Forte G. The enemy who sealed the world: Effects quarantine due to the COVID-19 on sleep quality, anxiety, and psychological distress in the Italian population. Sleep Med 2020; 75:12-20.

13. Lo K, Woo B, Wong M, Tam W. Subjective sleep quality, blood pressure, and hypertension: a meta-analysis. J Clin Hypertens (Greenwich) 2018; 20:592-605.

14. Ong AD, Kim S, Young S, Steptoe A. Positive affect and sleep: a systematic review. Sleep Med Rev 2017; 35:21-32.

15. Lima MG, Barros MBA, Ceolim MF, Zancanella E, Cardoso TAMO. Sleep duration, health and well-being: a population-based study. Rev Saúde Pública 2018; 52:82.

16. Faraut B, Boudjeltia KZ, Kerkhofs M. Immune, inflammatory and cardiovascular consequences of sleep restriction and recovery. Sleep Med Rev 2012; 16:137-49.

17. Chen Y, Zhao A, Lyu YXJ, Ye X. In the big picture of COVID-19 pandemic: what can sleep do. Sleep Med Rev 2012; 72:109-10. 
18. Gulia KK, Kumar VM. Importance of sleep for health and wellbeing amidst COVID-19 pandemic. Sleep Vigil 2020; [Epub ahead of print].

19. Becker PM. Sleep during times of coronavirus: early Chinese experience. Sleep Med 2020; [Epub ahead of print].

20. Sher L. COVID-19, anxiety, sleep disturbances and suicide. Sleep Med 2020; 70:124.

21. Cellini N, Canale N, Mioni G, Costa S. Changes in sleep pattern, sense of time and digital media use during COVID-19 lockdown in Italy. J Sleep Res 2020; 29:e13074.

22. Sandín B, Valiente RM, García-Escalera J, Chorot P. Impacto psicológico de la pandemia de COVID-19: efectos negativos y positivos en población española asociados al período de confinamiento nacional. Journal of Psychopathology and Clinical Psychology 2020; 25:122.

23. Szwarcwald CL, Souza Júnior PRB, Damacena GN, Malta DC, Barros MBA, Romero DE, et al. ConVid - Pesquisa de Comportamentos pela Internet durante a pandemia de COVID-19 no Brasil: concepção e metodologia de aplicação. Cad Saúde Pública 2021; 37:e00268320.

24. Xiao H, Zhang Y, Li S, Yang N. Social capital and sleep qu ality in individuals who self-isolated for 14 days during the coronavirus disease 2019 (COVID-19) outbreak in January 2020 in China. Med Sci Monit 2020; 26:e923921.

25. Whinnery J, Jackson N, Rattanaumpawan P, Grandner MA. Short and long sleep duration associated with race/ethnicity, sociodemographics, and socioeconomic position. Sleep 2014; 37:601-11.

26. Barros MBA, Lima MG, Ceolin MF, Zancanella E, Cardoso TAMO. Quality of sleep, health status and subjective well-being in a population-based study. Rev Saúde Pública 2019; 53:82.

27. Comisión Económica para América Latina y el Caribe. Panorama social de América Latina, 2019. https://repositorio.cepal.org/bitstream/ handle/11362/45090/1/S1900909_pt.pdf (acessado em 05/Jul/2020).

28. Kroth DC. A economia brasileira frente à pandemia do COVID-19: entre as prescrições e as propostas do governo. ResearchGate 2020; mar. https://www.researchgate. net/publication/340634459_A_ECONO MIA_BRASILEIRA_FRENTE_A_PAN DEMIA_DO_COVID-19_ENTRE_AS_PRES CRICOES_E_AS_PROPOSTAS_DO_GO VERNO (acessado em 06/Jul/2020).

29. Georges R. País estagnado: um retrato das desigualdades brasileiras. https://sinapse.gife. org.br/download/pais-estagnado-um-retra to-das-desigualdades-brasileiras (acessado em 06/Jul/20200).

30. Komatsu BK, Menezes-Filho N. Simulações de impactos da COVID-19 e da renda básica emergencial sobre o desemprego, renda, pobreza e desigualdade. https://www.insper.edu. br/wp-content/uploads/2020/04/Policy-Pa per-v14.pdf (acessado em 07/Jul/2020).
31. Zivin K, Paczkowski M, Galea S. Economic downturns and population mental health: research findings, gaps, challenges and priorities. Psychol Med 2011; 41:1343-8.

32. Karanikolos M, Mladovsky P, Cylus J, Thomson S, Basu S, Stuckler D, et al. Financial crisis, austerity, and health in Europe. Lancet 2013; 381:1323-31.

33. Martin-Carrasco M, Evans-Lacko S, Dom G. et al. EPA guidance on mental health and economic crises in Europe. Eur Arch Psychiatry Clin Neurosci 2016; 266:89-124.

34. Crew EC, Baron KG, Grandner MA, IeversLandis CE, McCrae CS, Nadorff MR, et al. The Society of Behavioral Sleep Medicine (SBSM) COVID-19 task force: objectives and summary recommendations for managing sleep during a pandemic. Behav Sleep Med 2020; [Online ahead of print].

35. Lima MG, Barros MBA, Alves MCGP. Sleep duration and health status self-assessment (SF36) in the elderly: a population-based study (ISA-Camp 2008). Cad Saúde Pública 2012; 28:1674-84.

36. Silva AA, Mello RGB, Schaan CW, Fuchs FD, Redline S, Fuchs SC. Sleep duration and mortality in the elderly: a systematic review with meta-analysis. BMJ Open 2016; 6:e008119.

37. Munhoz TN, Nunes BP, Wehrmeister FC, Santos IS, Matijasevich A. A nationwide population-based study of depression in Brazil. J Affect Disord 2016; 192:226-33.

38. Wang C, Pan R, Wan X, Tan Y, Xu L, Ho CS, et al. Immediate psychological responses and associated factors during the initial stage of the 2019 coronavirus disease (COVID-19) epidemic among the general population in China. Int J Environ Res Public Health 2020; 17:1729.

39. Pinho OS, Araújo TM. Associação entre sobrecarga doméstica e transtornos mentais comuns em mulheres. Rev Bras Epidemiol 2012; 15:560-72.

40. Hübscher M, Ferreira DML, Junqueira DGR, Refshauge KM, Maher CG, Hopper JL, et al. Heavy domestic, but not recreational, physical activity is associated with low back pain: Australian Twin low BACK pain (AUTBACK) study. Eur Spine J 2014; 23:2083-9.

41. Iguti AM, Bastos TF, Barros MBA. Dor nas costas em população adulta: estudo de base populacional em Campinas, São Paulo, Brasil. Cad Saúde Pública 2015; 31:2546-58.

42. Ohayon MM, Zulley J. Correlates of global sleep dissatisfaction in the German population. Sleep 2001; 24:780-7. 


\section{Abstract}

Sleep is a fundamental aspect for maintaining physical and emotional health, as well as one's well-being. Few studies have assessed the effect of socioeconomic conditions on sleep in the COVID-19 pandemic. Our objective was to analyze the increase or incidence of sleep disorders according to demographic and economic conditions, prior to the pandemic, and according to changes in financial, occupational, and household conditions during the pandemic. This study was conducted via web access, using data from April 24 to May 24 , with 45,160 Brazilians (aged 18 or older), with a sample weighted by Brazilian National Household Sample Survey (PNAD) data. Change in sleep quality (outcome), monthly income, effect on family income, occupation/work, gender, age group, marital status, and change in domestic work (exposures) were reported. The percentages of onset or increase of sleep disorders and adjusted odds ratio were estimated. The chance of exacerbation of sleep disorders was 34\%, 71\%, and twice as high in people with income less than one minimum wage before the pandemic, in those who lost their job and in those who had a great decrease in their income/ were without income, respectively. The chance of worsening sleep disorders was $82 \%$ higher in women; three times higher $(O R=3.14)$ in the population aged from 18 to 29, compared to the older adults; and higher with the increase in the amount of housework $(O R=2.21)$. Financial and occupational factors were determinants in the worsening of self-reported sleep quality, requiring rapid actions on these conditions in order to minimize this effect. Gender, age group, and household routines also deserve attention regarding sleep quality.

Sleep; Social Class; Coronavirus Infections

\section{Resumen}

El sueño es importante para mantener la salud física, emocional y bienestar. Pocos estudios evaluaron el efecto de las condiciones socioeconómicas en el sueño durante el período de la COVID-19. El objetivo fue analizar el aumento o incidencia de los problemas del sueño, según condiciones demográficas y económicas, previas a la pandemia, $y$ según cambios en las condiciones financieras, ocupacionales y tareas domésticas durante la pandemia. Estudio realizado vía web, usando datos del 24 de abril al 24 de mayo, con 45160 brasileños (18 o más), con una muestra ponderada por los datos de la Encuesta Nacional por Muestreo de Hogares (PNAD). Se informó de cambio en la calidad de sueño (desenlace), renta mensual, efecto en la renta familiar, en la ocupación/trabajo, sexo, franja etaria, situación conyugal y alteración en el trabajo doméstico (exposiciones). Estimamos los porcentajes de inicio o aumento de los problemas con el sueño y los OR ajustados. La oportunidad de exacerbación de los problemas con el sueño fue un $34 \%, 71 \%$ y 2 veces mayor en las personas con renta inferior a 1 salarios mínimos antes de la pandemia, en las que perdieron el empleo y en aquellas que tuvieron la renta muy disminuida/se quedaron sin renta, respectivamente. La oportunidad de empeorar los problemas de sueño fue un $82 \%$ mayor en las mujeres; tres veces mayor (OR = 3,14) en la población con 18 a 29 años, en relación con los ancianos; $y$ mayor con el incremento de la cantidad de tareas domésticas $(O R=2,21)$. Los factores financieros y ocupacionales fueron determinantes en el deterioro de la calidad del sueño autoinformada, demandando acciones rápidas sobre estas condiciones, a fin de minimizar este impacto. Género, franja de edad y rutinas domésticas también merecen atención en relación con la calidad del sueño.

Sueño; Clase Social; Infecciones por Coronavirus
Recebido em 23/Jul/2020

Versão final reapresentada em 17/Set/2020

Aprovado em 11/Dez/2020 